\title{
On the preparation of 1-aryl-2-heteroaryl- and 2-aryl-1-heteroaryl- pyrroles as useful building blocks for biologically interesting heterocycle
}

\author{
Anna Maria Almerico,* Alessandra Montalbano, Patrizia Diana, Paola Barraja, Antonino \\ Lauria, Girolamo Cirrincione, and Gaetano Dattolo \\ Dipartimento Farmacochimico, Tossicologico e Biologico Via Archirafi 32, 90123 Palermo, \\ Italy \\ E-mail: $\underline{\text { almerico@unipa.it }}$
}

\begin{abstract}
Dedicated to Professor Rudolph A. Abramovitch on the occasion of his $\mathbf{7 0}^{\text {th }}$ birthday (received 18 Jan 02; accepted 29 Jan 02; published on the web 06 Feb 02)
\end{abstract}

\begin{abstract}
A series of 1-aryl-2-pyridinyl/pyrimidinyl-pyrroles and 2-aryl-1-pyridinyl/pyrimidinyl-pyrroles were prepared by using 4+1 ring synthesis. The yields were strongly dependant on the reactivity of the starting amines. Synthetic procedures involving a 3+2 ring formation were discussed. Few 1-heteroaryl derivatives showed weak activity when tested as COX-1 and COX-2 inhibitors.
\end{abstract}

Keywords: 4+1 Pyrrole ring synthesis, 1-aryl-2-pyridinyl/pyrimidinyl-pyrroles, 2-aryl-1pyridinyl /pyrimidinyl-pyrroles, anti-inflammatory activity

\section{Introduction}

As our aim was devoted to study polycyclic nitrogen heterocycles we have been exploring synthetic pathways leading to the pyrrolo[1,2-f]phenanthridine ring system (PPH) to prepare derivatives with antineoplastic/antiviral activity. ${ }^{1-4}$ In preliminary biological screening tests, several PPHs have shown antiproliferative activity against cell lines derived from human tumors, sensible (FLC) and multidrug resistant (DRTL) with $\mathrm{IC}_{50}$ in the range 5-50 $\mu \mathrm{M}^{5}$ More recently they were demonstrated to possess a weak anti-HIV activity, joined to a capability of stimulating the multiplication of MT-4 cells at low concentrations. ${ }^{6}$

To further explore the biological features of related classes of planar molecules we decided to modify their carbon skeleton introducing the isosteric nitrogen. In particular the introduction of one or two nitrogens in the phenanthridine moiety could lead to several new heterocyclic systems annelated with the pyrrole ring, such as DiAzaPhenanthrenes (DAP) or TriAza-Phenanthrenes (TAP). To extend previously successful synthetic approaches for the preparation of PPHs we 
needed the access to large quantities of pyrrole derivatives of type $\mathbf{1}$ and $\mathbf{2}$, suitably substituted in the aryl/heteroaryl moiety.

These compounds should be useful starting materials for the synthesis of DAP and TAP which incorporate the pyrrole moiety, to be screened for their biological features. Moreover derivatives of type $\mathbf{1}$ and $\mathbf{2}$ themselves may be of interest in medicinal chemistry as they are related to 1,2-diaryl substituted pyrroles and pyrazoles that recently have been shown to posses interesting antiviral ${ }^{7}$ and anti-inflammatory ${ }^{8,9}$ activities.

However despite the apparent simplicity of the preparation of pyrroles of this type, some problems arise for their synthesis on a preparative scale as testified by the large number of papers dealing with pyrrole preparation. ${ }^{10,11}$ Moreover a literature survey indicated that whereas few 2pyridinyl-pyrroles have been reported, 2-pyrimidinyl-pyrroles of type 2 were unknown.

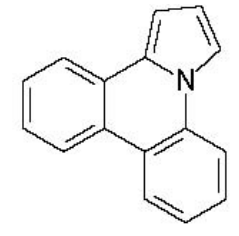

PPH
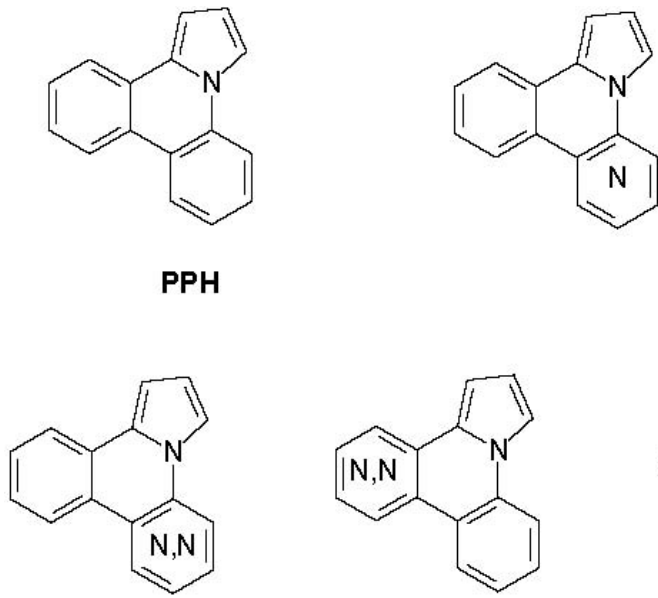

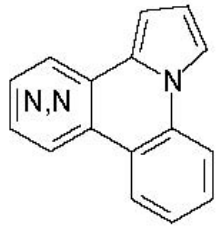

TAP
DAP

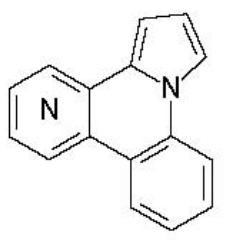<smiles>c1ccc(-n2cccc2)cc1</smiles>

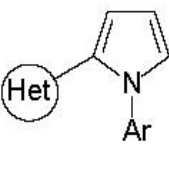

Het=PYRIDINE, PYRIMIDINE

\section{Results and Discussion}

In this paper we report our findings on the preparation of the title compounds exploring and comparing the synthetic routes leading to these compounds on a preparative scale on the basis of considerations about (a) low cost and ready availability of starting materials, (b) easy introduction of appropriate substituent in suitable positions of the rings, (c) reasonable yields. The experimental problems related to the synthesis of 1-heteroaryl or 2-heteroaryl-pyrroles are different and will be treated separately.

The preparation of 2-aryl-1-heteroarylpyrroles can be accomplished by a Paal-Knorr synthesis from 1,4-diketones of type $\mathbf{3}$ and commercially available aminopyridines / aminopyrimidines (Scheme 1). 

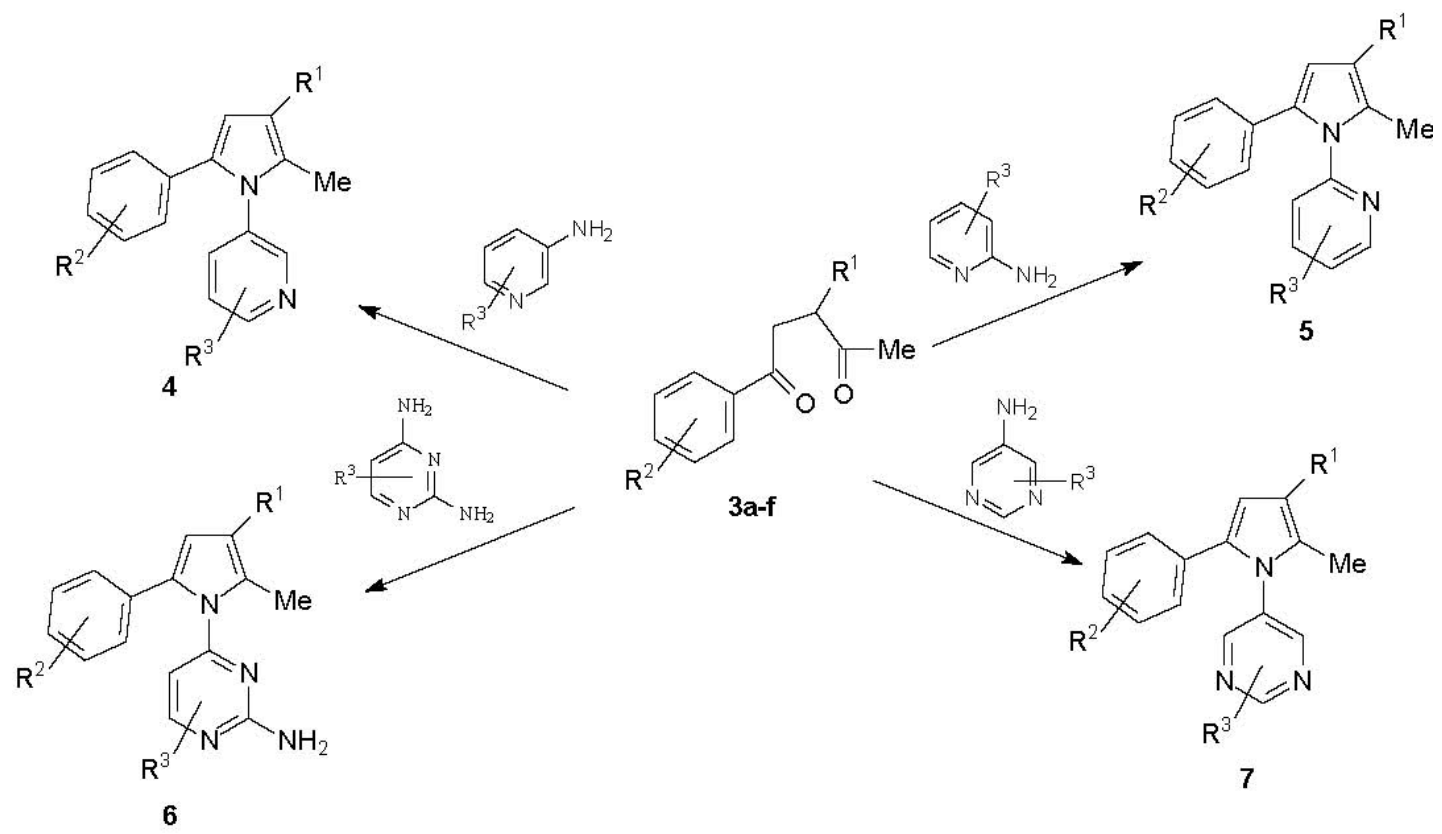

Scheme 1: $3 a, R^{1}=$ COOEt, $R^{2}=3-N_{2} ; 3 b, R^{1}=$ COOEt, $R^{2}=H ; 3 c, R^{1}=A c, R^{2}=H ; 3 d, R^{1}=A c$, $\mathrm{R}^{2}=2-\mathrm{NO}_{2} ; 3 \mathrm{e}, \mathrm{R}^{1}=\mathrm{Ac}, \mathrm{R}^{2}=3-\mathrm{OMe} ; 3 \mathrm{f}, \mathrm{R}^{1}=\mathrm{H}, \mathrm{R}^{2}=3-\mathrm{OMe}$.

In the case of derivatives of type $\mathbf{4}$ and $\mathbf{5}$, the yields were strongly dependent on the nucleophilicity of the starting amine. In fact, for example, pyrroles $\mathbf{4 g}$ and $\mathbf{4 h}$ were prepared in good yields whereas $5 \mathbf{j}$ and $5 \mathbf{l}$ were obtained only in $40-20 \%$ yields [together with the corresponding $\mathrm{N}$-acetylated derivatives $5 \mathbf{k}$ and $5 \mathbf{m}$ (yields $20 \%$ - traces) when the reaction was carried out in acetic acid]. By using diketone 3a and 2-aminopyridine a furan derivative, arising from intramolecular cyclization of the starting diketone, was always isolated and no traces of 1(pyridin-2-yl)pyrrole of type $5 \mathbf{i}$ could be detected.

In the case of aminopyrimidines it was possible to obtain pyrrole derivatives when the reacting amino group was in the 5 position of the starting pyrimidine (compounds of type 7) in yields ranging from 54 to $95 \%$. When the cyclization reactions were carried out on the less reactive 4 -amino derivatives only compound $\mathbf{6 n}$ was isolated in $20 \%$ yield, whereas compound 60 could not be obtained from the corresponding reactants. From the reaction of 1,4-diketone 3d and 4-amino-6-hydroxy-2-mercaptopyrimidine $\mathbf{8}$ unexpectedly a pyrido[2,3-d]pyrimidine derivative 9 was formed (Scheme 2). In this case the diketone 3d, being also a 1,3-dicarbonyl compound, preferentially reacted on the unsubstituted 5 position of the pyrimidine ring giving rise to a more stable bicyclic system. 
Table 1. 1-Heteroarylpyrrole derivatives and yields (\%)

\begin{tabular}{ccccc}
\hline Compounds & $\mathrm{R}^{1}$ & $\mathrm{R}^{2}$ & $\mathrm{R}^{3}$ & Yields \\
\hline $\mathbf{4 g}$ & $\mathrm{COOEt}$ & $3-\mathrm{NO}_{2}$ & $\mathrm{H}$ & 90 \\
$\mathbf{4 h}$ & $\mathrm{COOEt}$ & $3-\mathrm{NO}_{2}$ & $2,6-\mathrm{diOMe}$ & 60 \\
$\mathbf{5 i}$ & $\mathrm{COOEt}$ & $3-\mathrm{NO}_{2}$ & $\mathrm{H}$ & 0 \\
$\mathbf{5 j}$ & $\mathrm{COOEt}$ & $\mathrm{H}$ & $6-\mathrm{NH}_{2}$ & 40 \\
$\mathbf{5 k}$ & $\mathrm{COOEt}$ & $\mathrm{H}$ & $6-\mathrm{NHAc}$ & 20 \\
$\mathbf{5 l}$ & $\mathrm{Ac}$ & $\mathrm{H}$ & $6-\mathrm{NH}_{2}$ & 20 \\
$\mathbf{5 m}$ & $\mathrm{Ac}$ & $\mathrm{H}$ & $6-\mathrm{NHAc}$ & traces \\
$\mathbf{6 n}$ & $\mathrm{Ac}$ & $\mathrm{H}$ & $\mathrm{H}$ & 20 \\
$\mathbf{6 o}$ & $\mathrm{Ac}$ & $2-\mathrm{NO}_{2}$ & $6-\mathrm{OH}$ & 0 \\
$\mathbf{7 p}$ & $\mathrm{COOEt}$ & $3-\mathrm{NO}_{2}$ & $2,4-\mathrm{diOH}$ & 90 \\
$\mathbf{7 q}$ & $\mathrm{Ac}$ & $2-\mathrm{NO}_{2}$ & $2,4-\mathrm{diOH}$ & 54 \\
$\mathbf{7 r}$ & $\mathrm{Ac}$ & $3-\mathrm{OMe}^{-}$ & $4-\mathrm{NH}_{2}-6-\mathrm{OH}$ & 95 \\
$\mathbf{7 s}$ & $\mathrm{H}$ & $3-\mathrm{OMe}^{-}$ & $4-\mathrm{NH}_{2}-6-\mathrm{OH}$ & 70 \\
\hline
\end{tabular}<smiles>CC(=O)C(CC(=O)c1ccccc1[N+](=O)[O-])C(C)=O</smiles>

$3 d$<smiles>Nc1cc(O)nc(S)n1</smiles>

8<smiles>Cc1nc2nc(S)nc(O)c2c(C)c1CC(=O)c1ccccc1[N+](=O)[O-]</smiles>

9

Scheme 2

In the case of the synthesis of 1-aryl-2-heteroarylpyrroles the main problem is related to the accessibility of suitable 1,4-diketones or their synthetic equivalents. In fact for example the preparation of 2-pyridinyl-1-phenylpyrroles of type $\mathbf{1 0}$ was achieved so far according to the procedure outlined in the Scheme 3. Route $\mathrm{A}^{12}$ involved the condensation of pyridinyl derivatives of type $\mathbf{1 1}$ and aniline whereas in the case of route $\mathrm{B}^{13}$ the key intermediate was the oxazine 12, accessible in turn from 1,3-butadienes 13 and nitrosobenzene 14, which afforded the pyrrole $\mathbf{1 0}$ upon treatment with bases or acids. The yields obtained are listed in Table 2. 


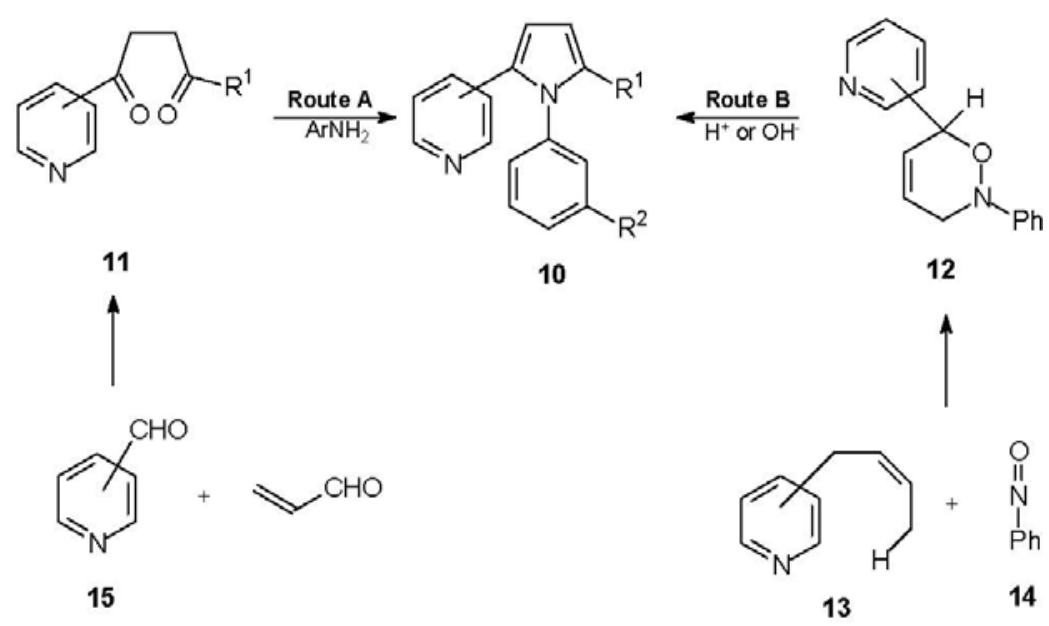

\section{Scheme 3}

Table 2. 2-Heteroarylpyrrole derivatives 10 and yields (\%)

\begin{tabular}{cccccc}
\hline Compounds & & $\mathrm{R}^{1}$ & $\mathrm{R}^{2}$ & Route & Yields \\
\hline $\mathbf{a}$ & pyridin-2-yl & $\mathrm{Me}$ & $\mathrm{H}$ & $\mathrm{A}$ & $\mathrm{nr}^{\mathrm{a}}$ \\
& & & & $\mathrm{B}$ & $86^{\mathrm{b}}$ \\
$\mathbf{b}$ & pyridin-2-yl & $\mathrm{Ph}$ & $\mathrm{H}$ & $\mathrm{A}$ & $\mathrm{nr}^{\mathrm{a}}$ \\
$\mathbf{c}$ & pyridin-3-yl & $\mathrm{Me}$ & $\mathrm{H}$ & $\mathrm{A}$ & $\mathrm{nr}^{\mathrm{a}}$ \\
$\mathbf{d}$ & pyridin-3-yl & $\mathrm{Ph}$ & $\mathrm{H}$ & $\mathrm{A}$ & $\mathrm{nr}^{\mathrm{a}}$ \\
$\mathbf{e}$ & pyridin-4-yl & $\mathrm{Me}$ & $\mathrm{H}$ & $\mathrm{A}$ & $\mathrm{nr}^{\mathrm{a}}$ \\
$\mathbf{f}$ & pyridin-2-yl & $\mathrm{H}$ & $\mathrm{H}$ & $\mathrm{B}$ & $93^{\mathrm{b}}$ \\
$\mathbf{g}$ & pyridin-3-yl & $\mathrm{H}$ & $\mathrm{H}$ & $\mathrm{B}$ & $96^{\mathrm{b}}$ \\
$\mathbf{h}$ & pyridin-4-yl & $\mathrm{H}$ & $\mathrm{H}$ & $\mathrm{B}$ & $98^{\mathrm{b}}$ \\
$\mathbf{i}$ & pyridin-2-yl & $\mathrm{H}$ & $\mathrm{NO}_{2}$ & $\mathrm{~A}$ & 40 \\
$\mathbf{j}$ & pyridin-3-yl & $\mathrm{H}$ & $\mathrm{NO}_{2}$ & $\mathrm{~A}$ & 40 \\
\hline
\end{tabular}

$\mathrm{nr}=$ not reported; ${ }^{\mathrm{a}}$ yields according to ref. 12 ; ${ }^{\mathrm{b}}$ yields according to ref 13.

Since our aim is devoted to the preparation of suitably substituted derivatives of type $\mathbf{1 0}$ we synthesized 2-pyridinylpyrroles $\mathbf{1 0} \mathbf{i}$ and $\mathbf{1 0 j}$, from 1,4-diketones $\mathbf{1 1}$ and 3-nitroaniline according to route A. The pyridinylketoaldehydes $\mathbf{1 1 i}$ and $\mathbf{1 1} \mathbf{j}$ (70-60\% yields) were prepared from the commercially available heterocyclic aldehydes $\mathbf{1 5}$ and acrolein in the presence of KCN.

However it was not possible to find commercially available pyrimidin-aldehydes and the synthetic procedure outlined by route A was not suitable for the preparation of 2pyrimidinylpyrroles. On the other hand attempts to prepare other diketones analogues of 11, by acylation of methylene active compounds with heterocyclic acyl bromides failed since it was impossible to obtain suitably substituted pyridin- or pyrimidin- $\mathrm{COCH}_{2} \mathrm{Br}$ from the corresponding acetyl derivatives. 
Therefore a different approach was undertaken involving a $3+2$ ring formation. In fact Katritzky and coworkers reported ${ }^{14}$ a new method for the synthesis of 1,2-diarylpyrroles 18 according to Scheme 4. This route employed as starting compounds the easy synthesizable Schiff bases, accessible in turn from aldehydes and amines. To verify if the reaction could be successful also in the case of heteroarylpyrroles, the imines $\mathbf{1 7}$ were prepared, generally in good yields (Table 3), and reacted according to literature procedure with 3-(benzotriazol-1-yl)-1-ethoxyprop1-ene 16.

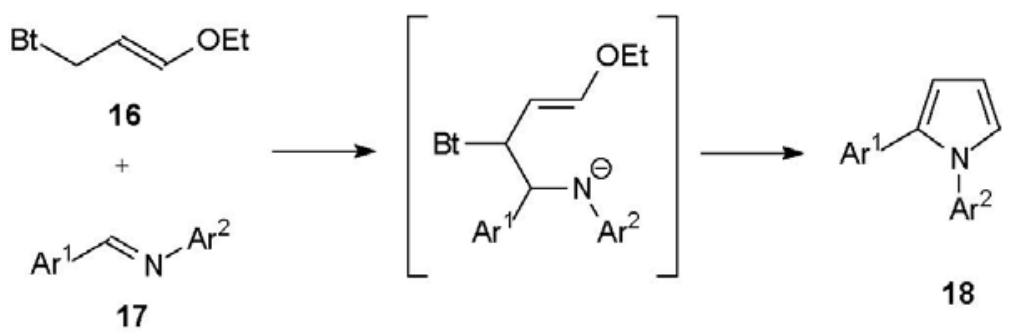

Scheme 4. $\mathrm{Bt}=$ benzotriazol-1-yl .

Table 3. Derivatives 17 and yields (\%)

\begin{tabular}{cccc}
\hline Compounds & $\mathrm{Ar}^{1}$ & $\mathrm{Ar}^{2}$ & Yields \\
\hline $\mathbf{a}$ & 3- $\mathrm{NO}_{2}-\mathrm{C}_{6} \mathrm{H}_{4}$ & pyridin-3-yl & 97 \\
b & 3- $\mathrm{NO}_{2}-\mathrm{C}_{6} \mathrm{H}_{4}$ & 6-OMe-pyridin-3-yl & 85 \\
c & pyridin-2-yl & 3- $\mathrm{NO}_{2}-\mathrm{C}_{6} \mathrm{H}_{4}$ & 40 \\
d & 3- $\mathrm{NO}_{2}-\mathrm{C}_{6} \mathrm{H}_{4}$ & 2-SH-6-OH-pyrimidin-4-yl & 69 \\
e & 3-OMe- $\mathrm{C}_{6} \mathrm{H}_{4}$ & 6- $\mathrm{NH}_{2}-4-\mathrm{OH}-$ pyrimidin-5-yl & 98 \\
\hline
\end{tabular}

From these reactions it was possible to isolate the pyrrole derivative 18 only when imine 17a was the starting material; in all the other cases extensive decomposition of the starting material was observed and intractable tars were mainly obtained. Therefore the method fails in the preparation of 2-pyrimidinyl-pyrroles that still remain unknown.

All the new 1-heteroarylpyrrole derivatives were proposed for a preliminary screening as anti-inflammatory agents. In fact it seems that the presence of aryl moieties, substituted with electron-withdrawing groups, in the positions 1 and/or 2 of the five membered ring is necessary for the appearance of the activity, as it was observed in the case of derivatives of type $19 .{ }^{9}$ 


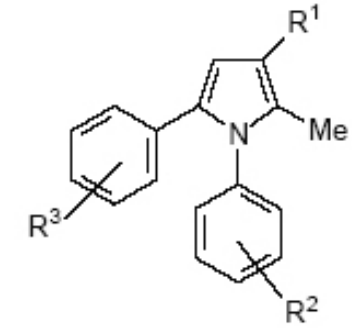

$19 \begin{aligned} & \mathrm{R}^{1}=\mathrm{H}, \mathrm{Ac}, \mathrm{CHO}, \mathrm{CN} \\ & \mathrm{R}^{2}=\mathrm{F}, \mathrm{CH}_{3}, \mathrm{Ac}, \mathrm{CF}_{3}, \mathrm{SO}_{2} \mathrm{Me} \\ & \mathrm{R}^{3}=\mathrm{F}, \mathrm{SO}_{2} \mathrm{Me}, \mathrm{SMe}\end{aligned}$<smiles>Cc1ccc(-c2cc(C(F)(F)F)nn2-c2ccc(S(N)(=O)=O)cc2)cc1</smiles>

CELECOXIB<smiles>[R]c1cccc(-c2cc([R])c(C)n2-c2cccc([R])c2)c1</smiles>

$20 \mathrm{R}^{1}=\mathrm{R}^{2}=\mathrm{H}, \mathrm{R}^{3}=\mathrm{NO}_{2}$ $21 \mathrm{R}^{1}=\mathrm{Ac}, \mathrm{R}^{2}=\mathrm{NH}_{2}, \mathrm{R}^{3}=\mathrm{OMe}$ $22 \mathrm{R}^{1}=\mathrm{Ac}, \mathrm{R}^{3}=\mathrm{N}_{3}, \mathrm{R}^{3}=\mathrm{OMe}$

This is even more evident in the case of the isoster pyrazole derivatives (i.e. celecoxib). However, in pyrrole series the structure-activity relationships are less straightforward although recently analogous behaviour was evidenced in the series of 3-aryl-4-aroyl compounds. ${ }^{8}$ Therefore we decided to include in the screening tests also some 1,2-diarylpyrroles, selected from our available database, such as compounds $\mathbf{2 0},{ }^{3} \mathbf{2 1},{ }^{4}$ and $22 .{ }^{4}$

The results of the screening tests for the 1-heteroarylpyrroles and related 1,2-diaryl derivatives 20-22 are reported in Table 4.

The only compounds which showed some activity against COX-1 at concentration 1-10 $\mu \mathrm{M}$ were 22, $4 \mathbf{g}$ and 7s, whereas derivative 7s weakly inhibited also COX-2. Therefore also in this case the presence of electron-withdrawing substituents or electron deficient rings seems necessary for the appearance of the activity.

Table 4. Inhibitory activity ${ }^{\mathrm{a}}(\%)$ of selected pyrroles

\begin{tabular}{|c|c|c|c|c|c|}
\hline Compound & Conc $(\mu \mathrm{M})$ & $\begin{array}{c}\mathrm{COX}-2 / \mathrm{PGG}_{2} \\
(\mathrm{ng} / \mathrm{mL})\end{array}$ & Inhib (\%) & $\begin{array}{c}\mathrm{COX}-1 / \mathrm{TXB}_{2} \\
(\mathrm{ng} / \mathrm{mL})\end{array}$ & Inhib (\%) \\
\hline \multirow[t]{3}{*}{20} & 0.1 & 23.622 & 0 & 763 & 4 \\
\hline & 1 & 27.446 & 0 & 695 & 13 \\
\hline & 10 & 35.850 & 0 & 711 & 11 \\
\hline \multirow[t]{3}{*}{21} & 0.1 & 32.813 & 0 & 843 & 0 \\
\hline & 1 & 28.053 & 0 & 652 & 18 \\
\hline & 10 & 33.410 & 0 & 732 & 8 \\
\hline \multirow[t]{3}{*}{22} & 0.1 & 31.513 & 0 & 570 & 28 \\
\hline & 1 & 30.418 & 0 & 582 & 27 \\
\hline & 10 & 41.798 & 0 & 720 & 10 \\
\hline \multirow[t]{3}{*}{$4 \mathrm{~g}$} & 0.1 & 32.647 & 0 & 769 & 4 \\
\hline & 1 & 32.647 & 0 & 765 & 4 \\
\hline & 10 & 48.283 & 0 & 575 & 28 \\
\hline
\end{tabular}


Table 4. Continued

\begin{tabular}{cccccc}
\hline $7 \mathbf{s}$ & 0.1 & 32.156 & 0 & 687 & 14 \\
& 1 & 15.997 & 16 & 623 & 22 \\
& 10 & 15.075 & 20 & 657 & 18 \\
\hline
\end{tabular}

${ }^{a}$ Expression and purification of human COX-1 and COX-2 enzymes and in vitro COX-1 and COX-2 enzyme assays were carried out according to reference 15. The inhibition of LPSinduced $\mathrm{PGG}_{2}$ production and $\mathrm{A} 23187$-induced $\mathrm{TBX}_{2}$ production in the human whole blood was studied utilizing the literature protocols. ${ }^{16,17}$

In conclusion from the data reported in this paper the best method for the preparation of heteroarylpyrroles remains the Paal-Knorr synthesis once the suitable 1,4-dicarbonyl compounds are available. 1-Heteroarylpyrroles constitute suitable model for the development of interesting biological compounds.

\section{Experimental Section}

General Procedures. Melting points (uncorrected) were taken on a Buchi-Tottoli capillary apparatus; IR spectra were determined in bromoform with a Jasco FT/IR 5300 spectrophotometer; ${ }^{1} \mathrm{H}$ and ${ }^{13} \mathrm{C}$ NMR spectra were measured at 200 and $50.3 \mathrm{MHz}$ respectively in $\left(\mathrm{CD}_{3}\right)_{2} \mathrm{SO}$ solution, unless otherwise specified, using a Bruker AC-E series $200 \mathrm{MHz}$ spectrometer (TMS as internal reference); mass spectra were obtained with a HP 5890 Series II and HP 5989A-GC/MS apparatus. Column chromatography was performed with Merck silica gel 230-400 Mesh ASTM or with a Biotage FLASH40i chromatography module (prepacked cartridge system). For all new compounds analyses indicated by the symbols of the elements or functions were within $\pm 0.4 \%$ of theoretical values.

Ethyl 2-methyl-5-(3-nitrophenyl)-1-(2,6-R ${ }^{3}$-pyridin-3-yl)-pyrrole-3-carboxylate (4g) and (4h). A solution of 1,4-diketone $3 \mathbf{a}^{3}(14 \mathrm{mmol})$ and 3-aminopyridine $(28 \mathrm{mmol})$ or 3-amino-2,6dimethoxypyridine $(14 \mathrm{mmol})$ in acetic acid was heated under reflux until disappearence of the starting diketone (TLC monitoring, $8 \mathrm{~h}$ and $3 \mathrm{~h}$ respectively). After cooling, the resultant solution was poured onto crushed ice. The precipitate was collected, air dried and recrystallized from ethanol.

4g. Yield 90\%, mp $135{ }^{\circ} \mathrm{C}$; IR v: $1697(\mathrm{CO}), 1531\left(\mathrm{NO}_{2}\right) \mathrm{cm}^{-1} ;{ }^{1} \mathrm{H}$ NMR $\left(\mathrm{CDCl}_{3}\right) \delta: 1.40(3 \mathrm{H}, \mathrm{t}$, $\left.J=7.2 \mathrm{~Hz}, \mathrm{CH}_{3}\right), 2.45\left(3 \mathrm{H}, \mathrm{s}, \mathrm{CH}_{3}\right), 4.33\left(2 \mathrm{H}, \mathrm{q}, J=7.2 \mathrm{~Hz}, \mathrm{CH}_{2}\right), 6.96(1 \mathrm{H}, \mathrm{s}$, pyrrole $\mathrm{H}-4)$, 7.30-7.36 $(2 \mathrm{H}, \mathrm{m}$, phenyl H-5 and H-6), $7.47(1 \mathrm{H}, \mathrm{dd}, J=8.0,4.5 \mathrm{~Hz}$, pyridine $\mathrm{H}-5), 7.61(1 \mathrm{H}$, d, $J=8.0 \mathrm{~Hz}$, pyridine $\mathrm{H}-4), 7.88(1 \mathrm{H}, \mathrm{d}, J=1.9 \mathrm{~Hz}$, phenyl H-2), $7.97(1 \mathrm{H}, \mathrm{dd}, J=7.2,1.9 \mathrm{~Hz}$, phenyl $\mathrm{H}-4), 8.47(1 \mathrm{H}, \mathrm{s}$, pyridine $\mathrm{H}-2), 8.69(1 \mathrm{H}, \mathrm{d}, J=4.5 \mathrm{~Hz}$, pyridine $\mathrm{H}-6) ;{ }^{13} \mathrm{C}$ NMR $\left(\mathrm{CDCl}_{3}\right) \delta: 12.5$ (q), 14.5 (q), 59.9 (t), 112.3 (d), 114.2 (s), 121.5 (d), 122.7 (d), 124.1 (d), 129.4 
(d), 131.5 (s), 133.3 (s), 133.6 (d), 134.2 (s), 135.8 (d), 139.2 (s), 148.1 (s), 149.2 (d), 149.9 (d), 164.9 (s). Anal $\left(\mathrm{C}_{19} \mathrm{H}_{17} \mathrm{~N}_{3} \mathrm{O}_{4}\right) \mathrm{C}, \mathrm{H}, \mathrm{N}$.

4h. Yield 60\%, mp $138{ }^{\circ} \mathrm{C}$; IR v: 1694 (CO), 1532 and $1346\left(\mathrm{NO}_{2}\right) \mathrm{cm}^{-1} ;{ }^{1} \mathrm{H}$ NMR $\delta: 1.38(3 \mathrm{H}, \mathrm{t}$, $\left.J=7.3 \mathrm{~Hz}, \mathrm{CH}_{3}\right), 2.35\left(3 \mathrm{H}, \mathrm{s}, \mathrm{CH}_{3}\right), 3.91\left(3 \mathrm{H}, \mathrm{s}, \mathrm{CH}_{3}\right), 3.96\left(3 \mathrm{H}, \mathrm{s}, \mathrm{CH}_{3}\right), 4.32(2 \mathrm{H}, \mathrm{q}, J=7.3$ $\left.\mathrm{Hz}, \mathrm{CH}_{2}\right), 6.32(1 \mathrm{H}, \mathrm{d}, J=8.0 \mathrm{~Hz}$, pyridine $\mathrm{H}-5), 6.90(1 \mathrm{H}, \mathrm{s}$, pyrrole $\mathrm{H}-4), 7.23(1 \mathrm{H}, \mathrm{d}, J=8.0$ $\mathrm{Hz}$, pyridine H-4), 7.35-7.45 (2H, m, phenyl H-6 and H-5), 7.96-8.00 (2H, m, phenyl H-2 and H4); ${ }^{13} \mathrm{C}$ NMR $\delta: 11.9$ (q), 14.5 (q), 53.8 (q), 53.9 (q), 59.6 (t), 101.9 (d), 111.1 (d), 112.4 (s), 113.2 (s), 121.2 (d), 122.3 (d), 129.0 (d), 131.7 (s), 133.3 (d), 134.1 (s), 140.1 (s), 140.8 (d), 148.0 (s), 158.4 (s), 163.1 (s), 165.1 (s). Anal $\left(\mathrm{C}_{21} \mathrm{H}_{21} \mathrm{~N}_{3} \mathrm{O}_{6}\right) \mathrm{C}, \mathrm{H}, \mathrm{N}$.

2-Methyl-5-phenyl-1-(6-R $\mathbf{R}^{\mathbf{3}}$-pyridin-2-yl)-pyrroles $\mathbf{5 j - m}$. A solution of 1,4 -diketones $3 \mathbf{b}^{18}$ or $3 \mathbf{c}^{19}$ (4.6 mmol) was heated under reflux with 2,6-diaminopyridine $(10 \mathrm{mmol})$ in acetic acid for $24 \mathrm{~h}$. Water was added and the aqueous layer was extracted with dichloromethane; the combined organic extracts were dried over sodium sulphate and the solvent was then removed under reduced pressure. The crude product was purified by column chromatography (dichloromethane/ethyl acetate 1:1).

In the case of the reaction with diketone $3 \mathbf{b}$, the first compound eluted was ethyl 1-(6aminopyridin-2-yl)-2-methyl-5-phenylpyrrole-3-carboxylate $5 \mathbf{j}$, yield $40 \%, \mathrm{mp} 210{ }^{\circ} \mathrm{C}$; IR v: 3385 and $3370\left(\mathrm{NH}_{2}\right), 1682(\mathrm{CO}) \mathrm{cm}^{-1}$; ${ }^{1} \mathrm{H} \mathrm{NMR} \delta: 1.29\left(3 \mathrm{H}, \mathrm{t}, J=7.2 \mathrm{~Hz}, \mathrm{CH}_{3}\right), 2.37(3 \mathrm{H}, \mathrm{s}$, $\left.\mathrm{CH}_{3}\right), 4.22\left(2 \mathrm{H}, \mathrm{q}, J=7.2 \mathrm{~Hz}, \mathrm{CH}_{2}\right), 6.31(1 \mathrm{H}, \mathrm{d}, J=7.3 \mathrm{~Hz}$, pyridine $\mathrm{H}-5), 6.38\left(2 \mathrm{H}, \mathrm{s}, \mathrm{NH}_{2}\right)$, $6.51(1 \mathrm{H}, \mathrm{d}, J=7.3 \mathrm{~Hz}$, pyridine $\mathrm{H}-3), 6.67(1 \mathrm{H}, \mathrm{s}$, pyrrole $\mathrm{H}-4), 7.07-7.28\left(5 \mathrm{H}, \mathrm{m}, \mathrm{C}_{6} \mathrm{H}_{5}\right), 7.51$ $\left(1 \mathrm{H}, \mathrm{t}, J=7.3 \mathrm{~Hz}\right.$, pyridine H-4); ${ }^{13} \mathrm{C} \mathrm{NMR} \delta: 11.9$ (q), 14.5 (q), 59.0 (t), 108.2 (d), 109.1 (d), 110.2 (d), 111.8 (s), 126.5 (s), 126.6 (d), 127.0 (d), 128.2 (d), 132.2 (s), 132.8 (s), 137.5 (s), 139.2 (d), 149.0 (s), 164.5 (s). Ms: m/z. $321\left(\mathrm{M}^{+}\right)$. Anal $\left(\mathrm{C}_{19} \mathrm{H}_{19} \mathrm{~N}_{3} \mathrm{O}_{2}\right) \mathrm{C}, \mathrm{H}, \mathrm{N}$.

The second compound eluted was ethyl 1-(6-acetylaminopyridin-2-yl)-2-methyl-5phenylpyrrole-3-carboxylate 5k, yield 20\%, mp $178{ }^{\circ} \mathrm{C}$; IR v: $3400(\mathrm{NH}), 1695$ (broad CO) $\mathrm{cm}^{-1}$; ${ }^{1} \mathrm{H}$ NMR $\delta: 1.30\left(3 \mathrm{H}, \mathrm{t}, J=7.2 \mathrm{~Hz}, \mathrm{CH}_{3}\right), 2.09\left(3 \mathrm{H}, \mathrm{s}, \mathrm{CH}_{3}\right), 2.37\left(3 \mathrm{H}, \mathrm{s}, \mathrm{CH}_{3}\right), 4.23(2 \mathrm{H}, \mathrm{q}, J=$ $\left.7.2 \mathrm{~Hz}, \mathrm{CH}_{2}\right), 6.70(1 \mathrm{H}, \mathrm{s}$, pyrrole $\mathrm{H}-4), 7.00-7.26\left(6 \mathrm{H}, \mathrm{m}, \mathrm{C}_{6} \mathrm{H}_{5}\right.$ and pyridine $\left.\mathrm{H}-3\right), 7.90(1 \mathrm{H}, \mathrm{t}, J$ $=8.8 \mathrm{~Hz}$, pyridine $\mathrm{H}-4), 8.20(1 \mathrm{H}, \mathrm{d}, J=8.8 \mathrm{~Hz}$, pyridine $\mathrm{H}-5) ;{ }^{13} \mathrm{C}$ NMR $\delta: 11.9$ (q), 14.4 (q), 23.9 (q), 59.2 (t), 109.5 (d), 112.3 (s), 113.3 (d), 118.4 (d), 126.8 (d), 127.2 (d), 128.4 (2d), 131.7 (s), 133.1 (s), 137.6 (s), 152.0 (s), 159.8 (s), 164.4 (s), 169.7 (s). Ms: m/z 363 (M ${ }^{+}$). Anal $\left(\mathrm{C}_{21} \mathrm{H}_{21} \mathrm{~N}_{3} \mathrm{O}_{3}\right) \mathrm{C}, \mathrm{H}, \mathrm{N}$.

In the case of the reaction with diketone 3c, the first compound eluted was 3-acetyl-1-(6aminopyridin-2-yl)-2-methyl-5-phenylpyrrole 5l, yield $20 \%, \mathrm{mp} 185{ }^{\circ} \mathrm{C}$; IR v: 3406 and 3333 $\left(\mathrm{NH}_{2}\right), 1649(\mathrm{CO}) \mathrm{cm}^{-1} ;{ }^{1} \mathrm{H}$ NMR $\delta: 2.47\left(3 \mathrm{H}, \mathrm{s}, \mathrm{CH}_{3}\right), 2.49\left(3 \mathrm{H}, \mathrm{s}, \mathrm{CH}_{3}\right), 4.76\left(2 \mathrm{H}, \mathrm{s}, \mathrm{NH}_{2}\right), 6.33$ $(1 \mathrm{H}, \mathrm{d}, J=7.3 \mathrm{~Hz}$, pyridine $\mathrm{H}-5), 6.46(1 \mathrm{H}, \mathrm{d}, J=7.3 \mathrm{~Hz}$, pyridine H-3), $6.68(1 \mathrm{H}$, s, pyrrole $\mathrm{H}-$ 4), 7.12-7.26 (5H, m, $\left.\mathrm{C}_{6} \mathrm{H}_{5}\right), 7.39(1 \mathrm{H}, \mathrm{t}, J=7.3 \mathrm{~Hz}$, pyridine $\mathrm{H}-4) ;{ }^{13} \mathrm{C}$ NMR $\delta: 12.8(\mathrm{q}), 28.8$ (q), 108.1 (d), 110.3 (d), 112.5 (d), 121.5 (s), 126.6 (d), 127.7 (d), 128.1 (d), 132.3 (s), 133.3 (s), 137.6 (s), 139.5 (d), 149.4 (s), 158.4 (s), 195.3 (s). Anal $\left(\mathrm{C}_{18} \mathrm{H}_{17} \mathrm{~N}_{3} \mathrm{O}\right) \mathrm{C}, \mathrm{H}, \mathrm{N}$.

The second compound eluted was 3-acetyl-1-(6-acetylaminopyridin-2-yl)-2-methyl-5- 
phenylpyrrole 5m [yield traces; Ms: m/z $333\left(\mathrm{M}^{+}\right)$].

3-Acetyl-1-(2-aminopyrimidin-4-yl)-2-methyl-5-phenylpyrrole (6n). A solution of 1,4diketone 3c (4.6 mmol) was heated under reflux with 2,4-diaminopyrimidine $(11.5 \mathrm{mmol})$ in acetic acid for $24 \mathrm{~h}$. After cooling, the reaction mixture was poured onto crushed ice and the insoluble dark residue was filtered off. The solution was then neutralized with $\mathrm{NaHCO}_{3}$ and extracted with dichloromethane; the combined organic extracts were dried over sodium sulphate and the solvent was then removed under reduced pressure to give an oil that solidified on addition of ethanol to give pyrrole $6 \mathbf{n}$, yield $20 \%$, mp $228^{\circ} \mathrm{C}$, IR v: 3320 and $3208\left(\mathrm{NH}_{2}\right), 1656$ (CO) $\mathrm{cm}^{-1} ;{ }^{1} \mathrm{H}$ NMR $\delta: 1.79\left(6 \mathrm{H}, \mathrm{s}, 2 \mathrm{xCH}_{3}\right), 6.31(1 \mathrm{H}, \mathrm{d}, J=6.8 \mathrm{~Hz}$, pyrimidine $\mathrm{H}-5), 6.95(2 \mathrm{H}$, $\left.\mathrm{s}, \mathrm{NH}_{2}\right), 7.28-7.36(4 \mathrm{H}, \mathrm{m}$, phenyl $\mathrm{H}-3, \mathrm{H}-4$ and $\mathrm{H}-5$, pyrrole $\mathrm{H}-4), 7.75(2 \mathrm{H}, \mathrm{d}, J=7.8 \mathrm{~Hz}$, phenyl H-2 and H-6), $8.12(1 \mathrm{H}, \mathrm{d}, J=6.8 \mathrm{~Hz}$, pyrimidine $\mathrm{H}-6)$. Anal $\left(\mathrm{C}_{17} \mathrm{H}_{16} \mathrm{~N}_{4} \mathrm{O}\right) \mathrm{C}, \mathrm{H}, \mathrm{N}$.

Ethyl 1-(2,4-dihydroxypyrimidin-5-yl)-2-methyl-5-(3-nitrophenyl)-pyrrole-3-car-boxylate (7p). Diketone 3a $(3.4 \mathrm{mmol})$ and 5-aminouracil $(3.4 \mathrm{mmol})$ in dry ethanol were heated under reflux for $21 \mathrm{~h}$. The solvent was evaporated under reduced pressure and the residue was purified by column chromatography using dichloromethane/ethyl acetate $1: 1$ as eluant. Yield 90\%, mp 132-133 ${ }^{\circ} \mathrm{C}$; IR v: 3554 (broad OH), 3250 (broad OH), $1690(\mathrm{CO}), 1545\left(\mathrm{NO}_{2}\right) \mathrm{cm}^{-1} ;{ }^{1} \mathrm{H}$ NMR $\delta$ : $1.29\left(3 \mathrm{H}, \mathrm{t}, J=6.8 \mathrm{~Hz}, \mathrm{CH}_{3}\right), 2.36\left(3 \mathrm{H}, \mathrm{s}, \mathrm{CH}_{3}\right), 4.23\left(2 \mathrm{H}, \mathrm{q}, J=6.8 \mathrm{~Hz}, \mathrm{CH}_{2}\right), 6.81(1 \mathrm{H}, \mathrm{s}$, pyrrole $\mathrm{H}-4), 7.61-7.75$ (2H, m, phenyl $\mathrm{H}-4$ and $\mathrm{H}-6), 7.97(1 \mathrm{H}, \mathrm{s}$, pyrimidine $\mathrm{H}-6), 8.04(1 \mathrm{H}, \mathrm{d}$, $J=3.9 \mathrm{~Hz}$, phenyl H-2), $8.11(1 \mathrm{H}, \mathrm{dt}, J=7.8,3.9 \mathrm{~Hz}$, phenyl H-5), $11.39(1 \mathrm{H}$, s, pyrimidine $\mathrm{OH}-4), 11.61$ (1H, s, pyrimidine $\mathrm{OH}-2) ;{ }^{13} \mathrm{C}$ NMR $\delta: 11.5$ (q), 14.4 (q), 59.1 (t), $110.4(\mathrm{~d}), 111.0$ (s), 112.4 (s), 121.6 (d), 121.7 (d), 130.1 (d), 132.0 (s), 133.3 (s), 133.9 (d), 140.0 (s), 143.6 (d), 147.7 (s), 150.6 (s), 161.2 (s), 164.1 (s). Anal $\left(\mathrm{C}_{18} \mathrm{H}_{16} \mathrm{~N}_{4} \mathrm{O}_{6}\right) \mathrm{C}, \mathrm{H}, \mathrm{N}$.

3-Acetyl-1-(2,4-dihydroxypyrimidin-5-yl)-2-methyl-5-(2-nitrophenyl)-pyrrole (7q). A suspension of o-nitrodiketone $3 \mathbf{d}^{20}(3,94 \mathrm{mmol})$ and 5-aminouracil $(3,94 \mathrm{mmol})$ was heated under reflux in dry ethanol for $12 \mathrm{~h}$. The pyrrole derivative $\mathbf{7 q}$ crystallized out from the solution on cooling. Yield 54\%, mp $>320{ }^{\circ} \mathrm{C}$; IR v: $3211(\mathrm{OH}), 1770(\mathrm{CO}), 1526\left(\mathrm{NO}_{2}\right) \mathrm{cm}^{-1} ;{ }^{1} \mathrm{H} \mathrm{NMR} \delta$ : $2.33\left(3 \mathrm{H}, \mathrm{s}, \mathrm{CH}_{3}\right), 2.36\left(3 \mathrm{H}, \mathrm{s}, \mathrm{CH}_{3}\right), 6.70(1 \mathrm{H}, \mathrm{s}$, pyrrole $\mathrm{H}-4), 7.40(1 \mathrm{H}, \mathrm{d}, J=7.5 \mathrm{~Hz}$, phenyl H-6), 7.53 (1H, s, pyrimidine H-6), 7.59-7.74 (2H, m, phenyl H-4 and H-5), 7.93 (1H, d, $J=7.5$ Hz, phenyl H-3); ${ }^{13} \mathrm{C}$ NMR $\delta: 12.0$ (q), 28.6 (q), 109.6 (s), 110.9 (d), 121.2 (s), 123.9 (d), 125.8 (d), 128.6 (s), 129.7 (d), 132.7 (d), 133.3 (d), 138.2 (s), 144.5 (s), 149.1 (s), 151.4 (s), 160.8 (s), 193.8 (s). Anal $\left(\mathrm{C}_{17} \mathrm{H}_{14} \mathrm{~N}_{4} \mathrm{O}_{5}\right) \mathrm{C}, \mathrm{H}, \mathrm{N}$.

1-(4-Amino-6-hydroxypyrimidin-5-yl)-5-(3-methoxyphenyl)-2-methyl-3- $\mathbf{R}^{\mathbf{1}}$-pyrroles $\mathbf{7 r}$ and 7s. The diketones $3 \mathbf{e}^{21}$ or $3 \mathbf{f}^{22}$ ( $\left.4 \mathrm{mmol}\right)$ dissolved in dry ethanol were added of 4,5-diamino-6hydroxypyrimidine $(4 \mathrm{mmol})$ and the suspension was heated under reflux in dry ethanol for $7 \mathrm{~h}$. The pyrrole derivative 7r, 7s crystallized out from the solution on cooling.

7r (yield 95\%), mp 223-225 ${ }^{\circ} \mathrm{C}$; IR v: $3412(\mathrm{OH}), 3316$ and $3281\left(\mathrm{NH}_{2}\right), 1643(\mathrm{CO}) \mathrm{cm}^{-1} ;{ }^{1} \mathrm{H}$ NMR $\delta: 2.25\left(3 \mathrm{H}, \mathrm{s}, \mathrm{CH}_{3}\right), 2.38\left(3 \mathrm{H}, \mathrm{s}, \mathrm{CH}_{3}\right), 3.68\left(3 \mathrm{H}, \mathrm{s}, \mathrm{CH}_{3}\right), 6.60\left(2 \mathrm{H}, \mathrm{bs}, \mathrm{NH}_{2}\right), 6.76(1 \mathrm{H}, \mathrm{d}$, $J=2.1 \mathrm{~Hz}$, phenyl H-2), 6.79 (1H, s, pyrrole H-4), $6.90(2 \mathrm{H}, \mathrm{dd}, J=8.6,2.1 \mathrm{~Hz}$, phenyl H-4 and H-6), 7.19 (1H, t, $J=8.6 \mathrm{~Hz}$, phenyl H-5), 7.88 (1H, s, pyrimidine $\mathrm{H}-2), 8.96(1 \mathrm{H}, \mathrm{bs}, \mathrm{OH}) ;{ }^{13} \mathrm{C}$ NMR $\delta: 11.4$ (q), 28.5 (q), 54.8 (q), 98.1 (s), 110.3 (d), 112.2 (d), 112.5 (d), 119.4 (d), 121.4 (s), 
129.1 (d), 133.1 (s), 133.7 (s), 137.8 (s), 149.3 (d), 158.8 (s), 158.8 (s), 160.5 (s), 193.7 (s). Anal $\left(\mathrm{C}_{18} \mathrm{H}_{18} \mathrm{~N}_{4} \mathrm{O}_{3}\right) \mathrm{C}, \mathrm{H}, \mathrm{N}$.

7s (yield 70\%), mp 250-255 ${ }^{\circ} \mathrm{C}$; IR v: 3398-3205 $\left(\mathrm{OH}\right.$ and $\left.\mathrm{NH}_{2}\right) \mathrm{cm}^{-1} ;{ }^{1} \mathrm{H}$ NMR $\delta: 1.97(3 \mathrm{H}, \mathrm{s}$, $\left.\mathrm{CH}_{3}\right), 3.66\left(3 \mathrm{H}, \mathrm{s}, \mathrm{CH}_{3}\right), 6.00(1 \mathrm{H}, \mathrm{d}, J=3.2 \mathrm{~Hz}$, pyrrole $\mathrm{H}-3), 6.27(1 \mathrm{H}, \mathrm{d}, J=3.2 \mathrm{~Hz}$, pyrrole $\mathrm{H}-4), 6.13\left(2 \mathrm{H}, \mathrm{bs}, \mathrm{NH}_{2}\right), 6.72(1 \mathrm{H}, \mathrm{dd}, J=7.5,2.1 \mathrm{~Hz}$, phenyl H-6), $6.83(1 \mathrm{H}, \mathrm{d}, J=2.1 \mathrm{~Hz}$, phenyl H-2), $6.88(1 \mathrm{H}, \mathrm{d}, J=7.5 \mathrm{~Hz}$, phenyl H-4), $7.17(1 \mathrm{H}, \mathrm{t}, J=7.5 \mathrm{~Hz}$, phenyl H-5), 7.87 (1H, s, pyrimidine H-2), $11.86(1 \mathrm{H}, \mathrm{s}, \mathrm{OH}) ;{ }^{13} \mathrm{C} \mathrm{NMR} \delta: 11.8$ (q), 54.7 (q), 99.6 (s), 107.4 (d), 108.5 (d), 111.5 (d), 111.5 (d), 118.8 (d), 129.0 (d), 131.4 (s), 133.0 (s), 134.8 (s), 148.9 (d), 158.9 (s), 159.3 (s), 160.8 (s). Anal $\left(\mathrm{C}_{16} \mathrm{H}_{16} \mathrm{~N}_{4} \mathrm{O}_{2}\right) \mathrm{C}, \mathrm{H}, \mathrm{N}$.

5,7-Dimethyl-4-hydroxy-2-mercapto-6-(2-nitrophenacyl)pyrido[2,3-d]pyrimidine (9). A suspension of 1,4-diketone 3d and 4-amino-3-hydroxy-2-mercaptopyrimidine $\mathbf{8}$ in dry ethanol $(60 \mathrm{~mL})$ was heated under reflux for $32 \mathrm{~h}$ in the presence of $p$-toluensulfonic acid $(0.26 \mathrm{mmol})$. After cooling of the reaction mixture, derivative 9 crystallized out from the solution. Yield 30\%, $\mathrm{mp}>290{ }^{\circ} \mathrm{C}$; IR v: $3283(\mathrm{OH}), 1697(\mathrm{CO}), 1548\left(\mathrm{NO}_{2}\right) \mathrm{cm}^{-1} ;{ }^{1} \mathrm{H}$ NMR $\delta: 2.52\left(3 \mathrm{H}, \mathrm{s}, \mathrm{CH}_{3}\right), 2.70$ $\left(3 \mathrm{H}, \mathrm{s}, \mathrm{CH}_{3}\right), 4.60\left(2 \mathrm{H}, \mathrm{s}, \mathrm{CH}_{2}\right), 7.93-7.98\left(4 \mathrm{H}, \mathrm{m}, \mathrm{C}_{6} \mathrm{H}_{4}\right), 12.39(1 \mathrm{H}, \mathrm{s}, \mathrm{OH}), 12.89(1 \mathrm{H}, \mathrm{s}, \mathrm{SH})$; ${ }^{13} \mathrm{C}$ NMR $\delta$ : 16.4 (q), 23.4 (q), 41.9 (t), 108.3 (s), 124.6 (d), 124.8 (s), 128.4 (d), 132.1 (d), 134.3 (d), 135.3 (s), 146.1 (s), 150.8 (s), 151.2 (s), 160.6 (s), 163.0 (s), 163.2 (s), 174.7 (s). Anal $\left(\mathrm{C}_{17} \mathrm{H}_{14} \mathrm{~N}_{4} \mathrm{O}_{4} \mathrm{~S}\right) \mathrm{C}, \mathrm{H}, \mathrm{N}, \mathrm{S}$.

4-Oxo-4-pyridinyl-butanal 11i and 11j. A solution of the appropriate pyridin-carboxyaldehyde (0.05 mmol) in anhydrous DMF was added dropwise to a mixture of sodium cyanide $(0.04 \mathrm{mmol})$ and anhydrous $\mathrm{DMF}$ at $0{ }^{\circ} \mathrm{C}$ under nitrogen. After 30 minutes acrolein $(0.05 \mathrm{mmol})$ in DMF was added dropwise. The mixture was stirred for $3 \mathrm{~h}$ at $0{ }^{\circ} \mathrm{C}$ and then acetic acid $(2.5 \mathrm{~mL})$ was added, followed 10 minutes later, by ice water. The $\mathrm{pH}$ was adjusted to 9 and the mixture was extracted with ethyl acetate; the combined organic extracts were dried over sodium sulphate and the solvent was then removed under reduced pressure. The residue was purified by chromatography from silica gel using ethyl acetate (11i) or dichloromethane/methanol 95:5 (11j) as eluent.

11i (yield 70\%), uncrystallizable oil; IR v: 1667 (broad CO) $\mathrm{cm}^{-1} ;{ }^{1} \mathrm{H}$ NMR $\left(\mathrm{CDCl}_{3}\right) \delta: 4.41-4.49$ $\left(2 \mathrm{H}, \mathrm{m}, \mathrm{CH}_{2}\right), 4.75-4.58\left(2 \mathrm{H}, \mathrm{m}, \mathrm{CH}_{2}\right), 7.15-7.25(1 \mathrm{H}, \mathrm{m}, \mathrm{H}-3), 7.41-7.48(1 \mathrm{H}, \mathrm{m}, \mathrm{H}-5), 7.59-$ 7.74 (2H, m, H-6 and H-4) 8.58-8.61 (1H, m, CHO). Ms: m/z $146\left(\mathrm{M}^{+}-17\right), 133,106,78$ (100\%). Anal $\left(\mathrm{C}_{15} \mathrm{H}_{11} \mathrm{~N}_{3} \mathrm{O}_{2}\right) \mathrm{C}, \mathrm{H}, \mathrm{N}$.

11j (yield 60\%), uncrystallizable oil; Ms: m/z $135\left(\mathrm{M}^{+}-28\right)$; this compound was immediately reacted without any further characterization.

1-(3-Nitrophenyl)-2-(pyridinyl)pyrroles $10 \mathbf{i}$ and $\mathbf{1 0 j}$. A solution of $11 \mathbf{i}$ or $\mathbf{1 1} \mathbf{j}(10 \mathrm{mmol})$ and 3-nitroaniline $(10 \mathrm{mmol})$ in dry ethanol with a catalytic amount of p-toluensulphonic acid, was heated under reflux for $7 \mathrm{~h}$ or $16 \mathrm{~h}$, respectively. The solvent was then removed under reduced pressure and the crude product was purified by column chromatography (dichloromethane/ethyl acetate $7: 3$, in the case of $\mathbf{1 0} \mathbf{i}, 95: 5$, in the case of $\mathbf{1 0} \mathbf{j})$.

$10 \mathbf{i}$ (yield 40\%), mp $125{ }^{\circ} \mathrm{C}$; IR v: $1524\left(\mathrm{NO}_{2}\right) \mathrm{cm}^{-1} ;{ }^{1} \mathrm{H} \mathrm{NMR}\left(\mathrm{CDCl}_{3}\right)$ 8: 7.10-7.28 (2H, m, pyrrole $\mathrm{H}-3$ and $\mathrm{H}-4), 7.46-7.96$ (5H, m, pyrrole $\mathrm{H}-5$; pyridine $\mathrm{H}-3$; phenyl H-5, H-6 and $\mathrm{H}-2$ ), 
$8.14(1 \mathrm{H}, \mathrm{d}, J=8.3 \mathrm{~Hz}$, phenyl H-4), 8.35-8.58 (2H, m, pyridine $\mathrm{H}-4$ and $\mathrm{H}-5), 8.95(1 \mathrm{H}, \mathrm{d}, J=$

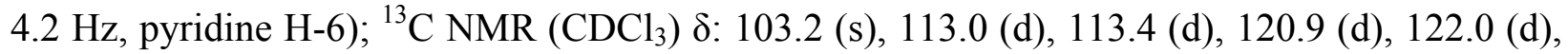
122.6 (d), 122.9 (d), 129.0 (d), 136.9 (d), 137.2 (d), 143.9 (s), 147.1 (d), 149.5 (d), 159.7 (s), 173.4 (s). Ms: m/z $265\left(\mathrm{M}^{+}\right), 218$. Anal $\left(\mathrm{C}_{15} \mathrm{H}_{11} \mathrm{~N}_{3} \mathrm{O}_{2}\right) \mathrm{C}, \mathrm{H}, \mathrm{N}$.

10j (yield 40\%), uncrystallizable oil; IR v: $1530\left(\mathrm{NO}_{2}\right) \mathrm{cm}^{-1} ;{ }^{1} \mathrm{H} \mathrm{NMR}\left(\mathrm{CDCl}_{3}\right) \delta: 6.52(1 \mathrm{H}, \mathrm{t}, J$ $=3.1 \mathrm{~Hz}$, pyrrole $\mathrm{H}-4), 6.70(1 \mathrm{H}, \mathrm{dd}, J=3.1,1.6 \mathrm{~Hz}$, pyrrole $\mathrm{H}-5), 7.11(1 \mathrm{H}, \mathrm{dd}, J=3.1,1.6 \mathrm{~Hz}$, pyrrole $\mathrm{H}-3), 7.42-7.70\left(5 \mathrm{H}, \mathrm{m}, \mathrm{C}_{6} \mathrm{H}_{4}\right.$ and pyridine $\left.\mathrm{H}-2\right), 8.12(1 \mathrm{H}, \mathrm{t}, J=3.3 \mathrm{~Hz}$, pyridine $\mathrm{H}-5)$, $8.24(1 \mathrm{H}, \mathrm{dd}, J=8.4,3.3 \mathrm{~Hz}$, pyridine $\mathrm{H}-4), 8.51(1 \mathrm{H}$, dd, $J=8.4,3.3 \mathrm{~Hz}$, pyridine $\mathrm{H}-6)$. Ms: $\mathrm{m} / \mathrm{z} 265\left(\mathrm{M}^{+}\right), 219$. Anal $\left(\mathrm{C}_{15} \mathrm{H}_{11} \mathrm{~N}_{3} \mathrm{O}_{2}\right) \mathrm{C}, \mathrm{H}, \mathrm{N}$.

$N$-(3-Nitrobenzylidene)pyridin-3-amine (17a). A solution of 3-nitrobenzaldehyde (6.6 mmol), 3 -aminopyridine $(6.6 \mathrm{mmol})$ and a catalytic amount of p-toluensulphonic acid was heated under reflux for $8 \mathrm{~h}$. The solvent was then removed under reduced pressure and the mixture was purified by column chromatography (dichloromethane/ethyl acetate 1:1). Yield 97\%, mp 105$110{ }^{\circ} \mathrm{C}$; IR v: $1630(\mathrm{CH}=\mathrm{N}), 1531\left(\mathrm{NO}_{2}\right) \mathrm{cm}^{-1} ;{ }^{1} \mathrm{H}$ NMR $\left(\mathrm{CDCl}_{3}\right) \delta: 7.37(1 \mathrm{H}, \mathrm{dd}, J=6.7,4.5$ $\mathrm{Hz}$, pyridine $\mathrm{H}-5), 7.59(1 \mathrm{H}, \mathrm{dt}, J=8.1,2.2 \mathrm{~Hz}$, phenyl H-6), $7.70(1 \mathrm{H}, \mathrm{t}, J=8.1 \mathrm{~Hz}$, phenyl H5), 8.27 (1H, dd, $J=6.7,1.5 \mathrm{~Hz}$, pyridine H-6), 8.35 (1H, dt, $J=8.1,2.2 \mathrm{~Hz}$, phenyl H-4), 8.49$8.57(2 \mathrm{H}, \mathrm{m}$, pyridine $\mathrm{H}-2$ and $\mathrm{H}-4), 8.58(1 \mathrm{H}, \mathrm{s}, \mathrm{CH}=\mathrm{N}), 8.76(1 \mathrm{H}, \mathrm{t}, J=2.2 \mathrm{~Hz}$, phenyl H-2); ${ }^{13} \mathrm{C}$ NMR $\delta: 123.5$ (d), 123.7 (d), 125.9 (d), 127.7 (d), 129.9 (d), 134.2 (d), 137.2 (s), 142.5 (d), 146.6 (s), 147.9 (d), 148.6 (s), 158.9 (d). Anal $\left(\mathrm{C}_{12} \mathrm{H}_{9} \mathrm{~N}_{3} \mathrm{O}_{2}\right) \mathrm{C}, \mathrm{H}, \mathrm{N}$.

6-Methoxy- $N$-(3-nitrobenzylidene)pyridin-3-amine (17b). 3-Nitrobenzaldehyde (18 mmol) and 5-amino-2-methoxypyridine $(18 \mathrm{mmol})$ were dissolved in dry ethanol $(100 \mathrm{~mL})$ and heated under reflux for $7 \mathrm{~h}$. After concentration under reduced pressure to a small volume, 17b crystallized from the reaction mixture. Yield 85\%, mp 90-92 ${ }^{\circ} \mathrm{C}$; IR v: $1624(\mathrm{CH}=\mathrm{N}), 1530$ $\left(\mathrm{NO}_{2}\right) \mathrm{cm}^{-1} ;{ }^{1} \mathrm{H} \mathrm{NMR}\left(\mathrm{CDCl}_{3}\right) \delta: 3.97\left(3 \mathrm{H}, \mathrm{s}, \mathrm{CH}_{3}\right), 6.80(1 \mathrm{H}, \mathrm{d}, J=8.8 \mathrm{~Hz}$, pyridine H-5), 7.58$7.69(1 \mathrm{H}, \mathrm{m}$, phenyl H-5), $7.65(1 \mathrm{H}, \mathrm{d}, J=7.8 \mathrm{~Hz}$, phenyl H-4), $8.18(1 \mathrm{H}, \mathrm{d}, J=8.8 \mathrm{~Hz}$, pyridine H-4), 8.20-8.31 (2H, m, phenyl H-2 and H-6), $8.57(1 \mathrm{H}, \mathrm{s}, \mathrm{CH}=\mathrm{N}), 8.71(1 \mathrm{H}, \mathrm{s}$, pyridine $\mathrm{H}-2)$; ${ }^{13} \mathrm{C}$ NMR $\delta: 53.6$ (q), 111.1 (d), 123.2 (d), 125.4 (d), 129.7 (d), 130.9 (d), 133.9 (d), 137.7 (s), 140.3 (d), 140.4 (s), 148.6 (s), 155.7 (d), 163.2 (s). Anal $\left(\mathrm{C}_{13} \mathrm{H}_{11} \mathrm{~N}_{3} \mathrm{O}_{3}\right) \mathrm{C}, \mathrm{H}, \mathrm{N}$.

$\boldsymbol{N}$-(3-Nitrophenyl)- $\boldsymbol{N}$-(pyridin-2-ylmethylene)amine (17c). A solution of pyridin-2carboxyaldehyde $(6.6 \mathrm{mmol}), 3$-nitroaniline $(6.6 \mathrm{mmol})$ and a catalytic amount of $\mathrm{p}$ toluenesulphonic acid, was heated under reflux in dry ethanol $(100 \mathrm{~mL})$ for $8 \mathrm{~h}$. The solvent was then removed in vacuo and the crude product was purified by column chromatography (dichloromethane/ethyl acetate 1:1). Yield 40\%, uncrystallizable oil; Ms: m/z $227\left(\mathrm{M}^{+}\right)$; this compound was immediately reacted without any further characterization.

6-[(3-Nitrobenzylidene)amino]-2-sulfanylpyrimidin-4-ol (17d). To a suspension of 3nitrobenzaldehyde $(6.6 \mathrm{mmol})$ and 4-amino-6-hydroxy-2-mercaptopyrimidine 8 (6.6 mmol) in dry ethanol $(100 \mathrm{~mL}) 5 \mathrm{~mL}$ of $\mathrm{HCl}$ in ethanol were added. The resulting solution was heated under reflux for $3 \mathrm{~h}$ and then cooled to room T. The resulting precipitate was filtered off and washed with warm $\mathrm{H}_{2} \mathrm{O}$. Yield $69 \%, \mathrm{mp}>290{ }^{\circ} \mathrm{C}$; IR v: $3408(\mathrm{OH}), 1553\left(\mathrm{NO}_{2}\right), 1636(\mathrm{CH}=\mathrm{N})$ $\mathrm{cm}^{-1} ;{ }^{1} \mathrm{H}$ NMR (pyridine-d $\left.{ }_{5}\right) \delta: 4.01(1 \mathrm{H}, \mathrm{s}, \mathrm{SH}), 6.27(1 \mathrm{H}, \mathrm{s}$, pyrimidine $\mathrm{H}-5), 7.48(1 \mathrm{H}, \mathrm{t}, J=$ 
$7.8 \mathrm{~Hz}$, phenyl H-5), 7.58 (1H, s, phenyl H-2), $7.82(1 \mathrm{H}, \mathrm{d}, J=7.8 \mathrm{~Hz}$, phenyl H-6), 8.11 (1H, d, $J=7.8 \mathrm{~Hz}$, phenyl H-4), $8.55(1 \mathrm{H}, \mathrm{s}, \mathrm{CH}=\mathrm{N}), 14.86(1 \mathrm{H}, \mathrm{bs}, \mathrm{OH}) ;{ }^{13} \mathrm{C} \mathrm{NMR} \delta: 121.1$ (d), 112.6 (d), 123.8 (d), 129.5 (d), 134.2 (d), 142.1 (s), 149.0 (s), 150.2 (d), 155.6 (s), 165.1 (s), 175.4 (s). Anal $\left(\mathrm{C}_{11} \mathrm{H}_{8} \mathrm{~N}_{4} \mathrm{O}_{3} \mathrm{~S}\right) \mathrm{C}, \mathrm{H}, \mathrm{N}, \mathrm{S}$.

6-Amino-5-[(3-methoxybenzylidene)amino]pyrimidin-4-ol (17e). To a solution of 3methoxybenzaldehyde $(7.93 \mathrm{mmol})$ in dry ethanol 4,5-diamino-6-hydroxypyrimidine (7.93 mmol) was added. The mixture was heated under reflux for $1 \mathrm{~h}$. On cooling a precipitate separated which was filtered off and washed with $\mathrm{H}_{2} \mathrm{O}$ at $60{ }^{\circ} \mathrm{C}$. Yield $98 \%$, mp 255-256 ${ }^{\circ} \mathrm{C}$; IR v: $3462(\mathrm{OH}), 3290$ and $3177\left(\mathrm{NH}_{2}\right), 1626(\mathrm{CH}=\mathrm{N}) \mathrm{cm}^{-1} ;{ }^{1} \mathrm{H}$ NMR $\delta: 3.83\left(3 \mathrm{H}, \mathrm{s}, \mathrm{CH}_{3}\right), 6.97$ $(1 \mathrm{H}, \mathrm{d}, J=7.5 \mathrm{~Hz}$, phenyl H-6), $6.99(2 \mathrm{H}, \mathrm{s} \mathrm{NH}), 7.32(1 \mathrm{H}, \mathrm{t}, J=7.5 \mathrm{~Hz}$, phenyl H-5), 7.43 $(1 \mathrm{H}, \mathrm{d}, J=7.5 \mathrm{~Hz}$, phenyl H-4), $7.51(1 \mathrm{H}, \mathrm{s}$, phenyl $\mathrm{H}-2), 7.76(1 \mathrm{H}, \mathrm{s}, \mathrm{CH}=\mathrm{N}), 9.84(1 \mathrm{H}, \mathrm{s}$, pyrimidine $\mathrm{H}-2), 11.83(1 \mathrm{H}, \mathrm{s}, \mathrm{OH}) ;{ }^{13} \mathrm{C} \mathrm{NMR} \delta: 55.2$ (q), 107.7 (s), 111.4 (d), 116.1 (d), 120.8 (d), 129.5 (d), 139.9 (s), 147.3 (d), 154.0 (d), 156.8 (s), 159.6 (s), 161.1 (s). Anal $\left(\mathrm{C}_{12} \mathrm{H}_{12} \mathrm{~N}_{4} \mathrm{O}_{2}\right)$ $\mathrm{C}, \mathrm{H}, \mathrm{N}$.

2-(3-Nitrophenyl)-1-(pyridin-3-yl)-pyrrole (18a). To a solution of $\mathbf{1 6}^{14}(5 \mathrm{mmol})$ in dry THF $(40 \mathrm{~mL})$ was added BuLi $(2 \mathrm{M}, 5.5 \mathrm{mmol}, 2.75 \mathrm{~mL})$ at $-78{ }^{\circ} \mathrm{C}$ under argon. After 30 minutes a solution of imine 17a $(5 \mathrm{mmol})$ in THF $(10 \mathrm{~mL})$ was added. The mixture was allowed to warm gradually to room $\mathrm{T}$ overnight. $\mathrm{ZnBr}_{2}(10 \mathrm{mmol})$ was added and the mixture was refluxed for $8 \mathrm{~h}$. Water $(150 \mathrm{~mL})$ was added and the mixture was extracted with diethyl ether. The combined organic layer was dried over sodium sulphate and the solvent was removed. The residue was subjected to column chromatography (dichloromethane/methanol 95:5). Yield 15\%, uncrystallizable oil; IR v: $1530\left(\mathrm{NO}_{2}\right) \mathrm{cm}^{-}{ }^{1} ;{ }^{1} \mathrm{H} \mathrm{NMR}\left(\mathrm{CDCl}_{3}\right) \delta: 6.83(1 \mathrm{H}, \mathrm{dd}, J=3.9,2.9 \mathrm{~Hz}$, pyrrole H-5), 7.06 (1H, dd, $J=4.9,3.9$, pyrrole $\mathrm{H}-4), 7.36$ (1H, dd, $J=4.9,2.9 \mathrm{~Hz}$, pyrrole H-3), $7.61(1 \mathrm{H}, \mathrm{t}, J=8.8 \mathrm{~Hz}$, phenyl H-5), $7.72(1 \mathrm{H}, \mathrm{d}, J=5.9 \mathrm{~Hz}$, pyridine H-5), $7.80(1 \mathrm{H}, \mathrm{d}, J=8.8$ $\mathrm{Hz}$, phenyl H-6), 8.03 (1H, d, $J=5.9 \mathrm{~Hz}$, pyridine $\mathrm{H}-4), 8.21(1 \mathrm{H}, \mathrm{d}, J=8.8 \mathrm{~Hz}$, phenyl H-4), $8.31\left(1 \mathrm{H}, \mathrm{s}\right.$, phenyl H-2), $8.40(1 \mathrm{H}, \mathrm{d}, J=5.9 \mathrm{~Hz}$, pyridine $\mathrm{H}-6), 8.77(1 \mathrm{H}$, s, pyridine $\mathrm{H}-2) ;{ }^{13} \mathrm{C}$ NMR $\left(\mathrm{CDCl}_{3}\right) \delta: 116.2$ (s), 120.2 (d), 121.2 (d), 121.7 (d), 122.4 (d), 123.7 (d), 126.4 (d), 128.3 (d), 129.9 (d), 130.4 (d), 132.5 (d), 133.9 (d), 145.5 (s), 148.8 (s), 164.3 (s). Anal $\left(\mathrm{C}_{16} \mathrm{H}_{13} \mathrm{~N}_{3} \mathrm{O}_{2}\right)$ C, H, N.

\section{References and Notes}

1. Dattolo, G.; Cirrincione, G.; Almerico, A. M.; Aiello E.; D’Asdia I. J. Heterocycl. Chem. 1986, 23, 1371.

2. Cirrincione, G.; Dattolo, G.; Almerico, A. M.; Presti, G.; Aiello, E. Heterocycles 1986, 24, 3403.

3. Almerico, A. M.; Cirrincione, G.; Dattolo, G.; Aiello, E.; Mingoia, F. J. Heterocycl. Chem. 1994, 31, 193.

4. Almerico, A. M.; Cirrincione, G.; Diana, P.; Grimaudo, S.; Dattolo, G.; Aiello, E.; Mingoia, 
F.; Barraja, P. Heterocycles 1994, 37, 1549.

5. Aiello, E.; Dattolo, G.; Cirrincione, G.; Almerico, A. M.; Diana, P.; Grimaudo, S.; Mingoia, F.; Barraja, P. Il Farmaco 1995, 50, 365.

6. Almerico, A. M.; Mingoia, F.; Diana, P.; Barraja, P.; Montalbano, A.; Lauria, A.; Loddo, R.; Sanna, L.; Delpiano, D.; Setzu, M. G.; Musiu, C. Eur. J Med. Chem. 2001, in the press.

7. Genin, M. J.; Biles, C.; Keiser, B. J.; Poppe, S. M.; Swaney, S. M.; Tarpley, W. G.; Yagi, Y.; Romero, D. L. J. Med. Chem. 2000, 43, 1034.

8. Dannhardt, G.; Kiefer, W.; Kramer, G.; Maehrlein, S.; Nowe, U.; Fiebich, B. Eur. J Med. Chem. 2000, 35, 499.

9. Khanna, I. K.; Weier, R. M.; Yu, Y.; Collins, P. W.; Miyashiro, J. M.; Koboldt, C. M.; Veenhuizen, A. W.; Currie, J. L.; Seibert, K.; Isakson, P. C. J. Med. Chem. 1997, 40, 1619.

10. see for example Cox, M.; Prager, R. H.; Riessen, D. M. ARKIVOC 2001, vii http://www.arkat-usa.org/ark/journal/Volume2/Part3/Cameron/cameron index.htm

11. Ketcha, D. M. In Progress in Heterocyclic Chemistry, Gribble, G. W.; Gilchrist, T. L. Eds, Pergamon, 2001; pp. 111-115.

12. Jones, R. A.; Karata M.; Voro, T. N.; Civeir, P. U.; Franck, A.; Ozturk, O.; Seaman, J. P.; Whitmore, A. P.; Williamson, D. J. Tetrahedron 1996, 52, 8707.

13. Stetter, H.; Schreckenberg, M. Chem. Ber. 1974, 107, 2453.

14. Katritzky, A. R.; Wu, H.; Xie, L.; Rachwal, S.; Rachwal, B.; Jiang, J.; Zhang, G.; Lang, H. Synthesis 1995, 1315.

15. Gierse, J. K.; Hauser, S. D.; Creely, D. P.; Koboldt, C.; Rangwala, S. H.; Isakson, P. C.; Seibert, K. Biochem. J. 1995, 305, 479.

16. Patrignani, P.; Panara, M. R.; Greco, A.; Fusco, O.; Natoli, C.; Iacobelli, S.; Cipollone, F.; Ganci, A.; Creminon, C.; Maclouf, J.; Patrono, C.J. Pharmacol. Exp. Ther. 1994, 271, 1705.

17. Brideau, C.; Kargman, S.; Liu, S.; Dallob, A. L.; Ehrich, E. W.; Rodger, I. W.; Chan, C.-C Inflamm. Res. 1996, 45, 68.

18. Paal, C. Chem. Ber. 1883, 16, 2867.

19. Aggarwal, J. S.; Qureshi, A. U.; Ray, J. N. J. Am. Chem. Soc. 1932, 54, 3988.

20. Aiello, E.; Dattolo, G.; Cirrincione, G.; Plescia, S.; Daidone, G. J. Heterocycl. Chem. 1979, $16,209$.

21. Dattolo, G.; Cirrincione, G.; Almerico, A. M.; Presti, G.; Aiello, E. Heterocycles 1984, 22, 2269.

This compound was obtained as uncrystallizable oil (yield 65\%) from diketone 3e upon heating under reflux for 30 min with $\mathrm{NaOH}$ ( $20 \%$ solution in ethanol). 